\title{
DNA Detection on a Power-free Microchip with Laminar Flow-assisted Dendritic Amplification
}

\author{
Kazuo Hosokawa, ${ }^{\dagger}$ Takahiro SATo, Yasunobu SATo, and Mizuo MaEda \\ Bioengineering Laboratory, RIKEN, 2-1 Hirosawa, Wako, Saitama 351-0198, Japan
}

\begin{abstract}
In this paper, we describe DNA detection experiments using our two original technologies, power-free microchip and laminar flow-assisted dendritic amplification (LFDA), which were previously applied to immunoassays. A microchip was fabricated by combining a poly(dimethylsiloxane) (PDMS) part having microchannel patterns and a glass plate modified with probe DNA. We carried out two kinds of experiments: the detection of 21-base biotinylated target DNA and the detection of single-nucleotide polymorphism (SNP) in 56-base unlabeled target DNA by sandwich hybridization with biotinylated probe DNA. For both of the experiments, the necessary solutions were injected into microchannels not by an external power source, but by air dissolution into the PDMS part. After a hybridization reaction, the LFDA was started by injecting FITC-labeled streptavidin and biotinylated anti-streptavidin antibody onto the reaction site. With a detection time of $20 \mathrm{~min}$, the limit of detection (LOD) for the biotinylated target was $2.2 \mathrm{pM}$, and the LOD for the SNP was 10 - $30 \mathrm{pM}$, depending on the SNP type.
\end{abstract}

(Received July 8, 2010; Accepted August 7, 2010; Published October 10, 2010)

\section{Introduction}

DNA detection plays an important role in almost every field of modern life science and technology. Especially, the detection of single-nucleotide polymorphism (SNP), which is the most common form of genetic variation, ${ }^{1}$ is routinely performed for genomics studies, the diagnosis of monogenetic diseases, and forensic examinations. One of the most important technologies for DNA detection is hybridization on solid-phase supports, which forms the principle of microarrays. In fact, modern microarrays enable the detection of millions of SNPs in a single assay, ${ }^{2}$ and a specialized amplification technique has realized a limit of detection (LOD) as low as $50 \mathrm{fM}^{3}$ However, one drawback of solid-phase hybridization is the long incubation time (at least $1-2 \mathrm{~h}$ and often overnight), which is required as a result of the diffusion-limited reaction kinetics. This drawback makes solid-phase hybridization difficult for point-of-care (POCT) applications.

To overcome this drawback, the use of microfluidic devices (microchips; we use this term only for devices with microchannels in the current work, and not for conventional microarrays) is an attractive choice, because it is widely accepted that the use of microchips reduces the time of the solid-phase hybridization assays. This effect is attributable to a shortening of the distance that the DNA molecules must travel to the solid-phase support by diffusion. ${ }^{4}$ The same effect occurs with microchip immunoassays. ${ }^{5}$ There have been numerous papers reporting solid-phase hybridization assays on microchips with varying degrees of success. ${ }^{6-12}$ The common advantage of these devices is the short assay time, typically $10-30 \mathrm{~min}$. However, there is still much room for improvements, especially

$\dagger$ To whom correspondence should be addressed.

E-mail: k-hoso@riken.go.jp concerning two issues: simplicity and sensitivity. Most of the previous microchips required external apparatuses for fluid pumping: pressure sources for pressure-driven flow, ${ }^{6-9}$ high-voltage power supplies for electrokinetic flow, ${ }^{10}$ and mechanical rotators for centrifugation-induced flow. ${ }^{11,12}$ Such an apparatus considerably raises the system's complexity, which may become a major obstacle to achieve POCT applications. The other issue for improvement is sensitivity. The existing microchips for solid-phase hybridization assays generally showed an LOD higher than sub-nanomolar, ${ }^{6,710-12}$ with few exceptions. ${ }^{8,9}$

Recently, we developed a simple and sensitive method for microchip solid-phase immunoassays, ${ }^{13}$ which share the fundamental principle with solid-phase hybridization assays. Simplicity was achieved by a power-free pumping technique, ${ }^{14}$ which eliminated the need for external power sources for fluid pumping. With this technique, we were able to inject the samples and reagents into the microchannels only by dispensing (placing) droplets of the solutions at the inlet openings of the microchannels. The sensitivity was enhanced by a new signal amplification method: laminar flow-assisted dendritic amplification (LFDA). In this method, two amplification reagents, FITC-labeled streptavidin (F-SA) and biotinylated anti-streptavidin (B-anti-SA), were supplied from different microchannel branches to a common microchannel where a biotinylated secondary antibody had been bound to the channel wall via an analyte. The use of laminar flow enabled a rapid and continuous growth of aggregates of the F-SA and B-anti-SA molecules on the analyte molecules. As a result, we were able to detect $0.15 \mathrm{pM}$ C-reactive protein with a detection time of 23 min. ${ }^{13}$ These results motivated us to apply the power-free microchip and the LFDA to solid-phase hybridization assays as well, because of the shared fundamental principle.

In this paper, we report on the successful application of the power-free microchip and LFDA to DNA detection through 
Table 1 Sequences of the oligonucleotides used in this work

\begin{tabular}{llc}
\hline Symbol & \multicolumn{1}{c}{ Sequence $^{\mathrm{a}, \mathrm{b}}$} & $T_{\mathrm{m}}{ }^{\circ}{ }^{\circ} \mathrm{C}$ \\
\hline CP & $\begin{array}{l}\mathrm{NH}_{2} \text {-TTT TTT TTT TTT TTT AAG AGA CCG TTG } \\
\text { GGG CGA AAG }\end{array}$ & 59.5 \\
B-tar & $\begin{array}{l}\text { biotin-CTT TCG CCC CAA CGG TCT CTT } \\
\text { B-ran }\end{array}$ & biotin-TTG TTA TGG GTT CTC \\
WT & ACC CCT TAC CCG CAT CTC CCA CCC CCA GGA \\
\multicolumn{3}{c}{ CGC CCC TTT CGC CCC AAC GGT CTC TT } \\
MT & ACC CCT TAC CCG CAT CTC CCA CCC CCA AGA \\
& CGC CCC TTT CGC CCC AAC GGT CTC TT \\
BP-WT CGT CCT GGG G-biotin & 42.7 \\
BP-MT CGT CTTT GGG G-biotin & 38.6 \\
\hline
\end{tabular}

a. Written from $5^{\prime}$ to $3^{\prime}$.

b. The underlines indicate SNP bases.

c. Calculated with the GC\% method.

solid-phase hybridization. We first tried preliminary experiments in which biotinylated 21-base target DNA was detected. We were able to detect the biotinylated target at concentrations down to $2.2 \mathrm{pM}$ in $20 \mathrm{~min}$. Next, we tried the detection of a SNP in unlabeled 56-base target DNA by sandwich hybridization. In this case, the LOD was $10-30 \mathrm{pM}$, and the detection time was also $20 \mathrm{~min}$.

\section{Experimental}

\section{Reagents and chemicals}

Table 1 lists the sequences of the oligonucleotides used in this work. These sequences were basically adopted from our previous work, ${ }^{15}$ in which sandwich-type affinity electrophoresis was carried out on a microchip. Namely, wild type (WT) and mutant type (MT) targets were designed to simulate a human gene, CYP2D6 (dbSNP ID: rs3892097). A capture probe (CP) was designed to hybridize to the $3^{\prime}$ region of WT and MT, and the $(\mathrm{dT})_{15}$ sequence was attached to CP to alleviate any possible steric hindrance caused by the solid support. A biotinylated probe for WT (BP-WT) and a biotinylated probe for MT (BP-MT) were designed to hybridize to the SNP parts of WT and MT, respectively. A biotinylated target (B-tar) is a partial sequence of WT which hybridizes to $\mathrm{CP}$. Biotinylated random DNA (B-ran) was originally designed for different research, but it was used to confirm the specificity of detection of B-tar in this work.

WT and MT were synthesized and HPLC-purified by Operon Biotechnologies (Tokyo, Japan). All of the other oligonucleotides were synthesized and HPLC-purified by Tsukuba Oligo Service (Ushiku, Japan). 3-Glycidoxypropyltrimethoxysilane (GPTMS) was obtained from Shinetsu Chemcals (Tokyo, Japan). Casein-based blocking reagent (BR) was obtained from Roche Diagnostics (Manheim, Germany). FITC-labeled streptavidin (F-SA) was obtained from Sigma (St. Louis, MO). Biotinylated anti-streptavidin (B-anti-SA) was obtained from Rockland Immunochemicals (Gilbertsville, PA). Other chemicals were obtained from Wako Pure Chemical Industries (Osaka, Japan). Deionized (DI) water was produced with a Milli-Q SP system (Nihon Millipore, Tokyo, Japan).

\section{Microchip fabrication}

We designed and fabricated a poly(dimethylsiloxane) (PDMS)-glass hybrid microchip, shown in Fig. 1. The microchip has two sets of microchannels with the double-Y (a)

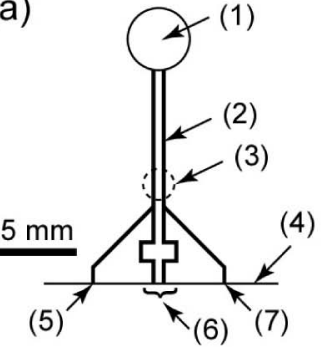

(b)

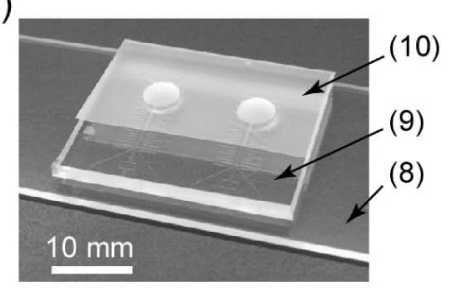

Fig. 1 (a) Microchannel design: (1) waste reservoir, (2) microchannel, (3) CP spot on the glass surface, (4) PDMS edge, (5) left inlet, (6) center inlets, (7) right inlet. The microchannels were $100 \mu \mathrm{m}$ wide and $25 \mu \mathrm{m}$ deep. (b) Fabricated microchip: (8) glass plate, (9) PDMS part, (10) adhesive tape.

geometry shown in Fig. 1a. The detours of the center channels were constructed to make the fluidic resistances (proportional to the channel length) uniform.

First, the PDMS part with recessed patterns of the microchannels was fabricated as described elsewhere. ${ }^{14}$ Briefly, a negative master for the molding of PDMS was fabricated on a silicon wafer with an ultrathick photoresist (SU-8 25, MicroChem, Newton, MA). The surface of the master was passivated by exposure to $\mathrm{CHF}_{3}$ plasma in a plasma etcher (RIE-10NR, Samco International, Kyoto, Japan). Prepolymer of PDMS (Sylgard 184, Dow Corning, Midland, MI) was cast onto the master with a frame for holding the prepolymer. The cured PDMS part was peeled off from the master, and thorough-holes for the waste reservoirs were punched using a metal pipe. To make the inlet openings, the PDMS part was cut with a knife.

Next, CP was covalently immobilized on a glass plate with the epoxy-amine coupling method. ${ }^{16}$ Briefly, a $76 \times 26 \times 1 \mathrm{~mm}$ slide glass (Matsunami Glass, Osaka, Japan) was immersed in a $10 \%$ sodium hydroxide solution at room temperature overnight. After rinsing with a $10 \%$ hydrogen chloride solution, the glass plate was washed with DI water three times. The residual water on the glass plate was blown off by a nitrogen stream, and then the glass plate was dried in an oven (AWC-2, TGK, Tokyo, Japan) at $90^{\circ} \mathrm{C}$ for $15 \mathrm{~min}$. The dried glass plate was immersed in a $0.1 \%$ GPTMS solution in dehydrated toluene at room temperature for $1 \mathrm{~h}$. The glass plate was sequentially washed with the following solvents: toluene, $50 \%$ toluene in ethanol, ethanol, and DI water. The residual water was blown off, and then the glass plate was dried in the oven at $110^{\circ} \mathrm{C}$ for $30 \mathrm{~min}$. A $25 \mu \mathrm{M}$ CP solution in $0.15 \mathrm{M}$ phosphate buffer ( $\mathrm{pH}$ 7.4) was prepared, and two aliquots of $2.0 \mu \mathrm{L}$ of the $\mathrm{CP}$ solution were spotted at proper positions onto the glass plate using a micropipette. The proper positioning was achieved by placing the glass plate onto a paper sheet on which the target positions were marked. The glass plate was immediately put into a humidified box, which was then placed in an incubator (CTU-N, Taitec, Koshigaya, Japan) at $40^{\circ} \mathrm{C}$ for $12 \mathrm{~h}$. The glass surface was washed with DI water, and dried under a nitrogen stream.

Finally, the PDMS part was placed onto the glass plate so that the microchannels overlapped the $\mathrm{CP}$ spots. The PDMS part and the glass plate were reversibly bonded to each other.

\section{Direct detection of biotinylated target}

A blocking solution was prepared by dissolving $1 \%$ BR and $0.02 \%$ sodium dodecyl sulfate (SDS) in a $5 \times$ saline-sodium citrate (SSC) buffer (pH 7.0). Samples and LFDA reagents (F-SA and B-anti-SA solutions) were prepared by dissolving the 
following components in the blocking solution: 0 - $10 \mathrm{nM}$ B-tar and $1.0 \mu \mathrm{M}$ B-ran for the samples, $2.5 \mu \mathrm{g} / \mathrm{mL}$ F-SA for the F-SA solution, and $25 \mu \mathrm{g} / \mathrm{mL}$ B-anti-SA for the B-anti-SA solution.

The samples and reagents were injected into the microchannels with the power-free pumping technique. ${ }^{14}$ Briefly, the microchip was degassed in a vacuum desiccator at $10 \mathrm{kPa}$ for $1 \mathrm{~h}$. Immediately after removal from the desiccator, the upper face of the PDMS was covered with a piece of adhesive tape. The microchip was placed on the stage of an inverted fluorescence microscope (TE2000-U, Nikon, Tokyo, Japan). A $0.5-6.0 \mu \mathrm{L}$ aliquot of the solution to be injected was dispensed at the inlet specified below with a micropipette. The experiments were carried out at room temperature $\left(\sim 25^{\circ} \mathrm{C}\right)$ using freshly fabricated microchips.

The injection sequence was as follows: (Step 1, blocking for $5 \mathrm{~min}$ ) $0.5 \mu \mathrm{L}$ blocking solution to the left inlet, $1.0 \mu \mathrm{L}$ blocking solution to the center inlets, and $0.5 \mu \mathrm{L}$ blocking solution to the right inlet; (Step 2, hybridization for $5 \mathrm{~min}$ ) $0.5 \mu \mathrm{L}$ of $0.10 \mathrm{pM}$ $10 \mathrm{nM}$ B-tar sample to the left, $1.0 \mu \mathrm{L}$ blocking solution to the center, and $0.5 \mu \mathrm{L}$ blank sample (containing $1.0 \mu \mathrm{M}$ B-ran only) to the right; and (Step 3, LFDA for $15 \mathrm{~min}$ ) $3.0 \mu \mathrm{L}$ B-anti-SA solution to the left, $6.0 \mu \mathrm{L} \mathrm{F-SA}$ solution to the center, and $3.0 \mu \mathrm{L}$ B-anti-SA solution to the right.

\section{SNP detection of unlabeled targets}

The blocking solution and the LFDA reagents (F-SA and B-anti-SA solutions) were the same as those mentioned in the previous section. Samples (WT and MT) and biotinylated probe solutions (BP-WT and BP-MT) were prepared by dissolving the following components in the blocking solution: $0-10 \mathrm{nM}$ MT or WT for the samples, and $1.0 \mu \mathrm{M}$ BP-WT or BP-MT for the biotinylated probe solution.

The samples and reagents were injected into the microchannels using the same technique as described in the previous section. The experiments were carried out at room temperature $\left(\sim 25^{\circ} \mathrm{C}\right)$ using freshly fabricated microchips. The injection sequence was also the same as that mentioned in the previous section, except that Step 2 was changed as follows: (Step 2, hybridization for $5 \mathrm{~min}$ ) $0.5 \mu \mathrm{L}$ BP-WT solution to the left inlet, $1.0 \mu \mathrm{L} \mathrm{WT}$ or MT sample to the center inlets, and $0.5 \mu \mathrm{L}$ BP-MT solution to the right inlet.

\section{Data acquisition and analysis}

During the LFDA step, fluorescence images of the microchannels at the $\mathrm{CP}$ spot were taken every 1 min using the inverted fluorescence microscope equipped with a 100-W mercury lamp, a dichroic mirror block (excitation 465 - $495 \mathrm{~nm}$ and emission 515 - $555 \mathrm{~nm}$ ), a 10× objective lens, and a cooled CCD camera (CoolSNAP HQ2, Photometrics, Tucson, AZ). The exposure time of the CCD camera was varied between 0.5 and $1.0 \mathrm{~s}$ so as to avoid signal saturation. An intensity profile plot across the microchannels at $250-350 \mu \mathrm{m}$ downstream from the confluent points was obtained using image analysis software (Image J 1.38x, National Institutes of Health, USA). The data were normalized by subtracting the background intensity at the outside of the channels and by dividing the remainder by the exposure time of the CCD camera.

\section{Results and Discussion}

We carried out two kinds of DNA detection experiments: the direct detection of a biotinylated target DNA (Fig. 2b) and the SNP detection of an unlabeled target DNA by sandwich (a)
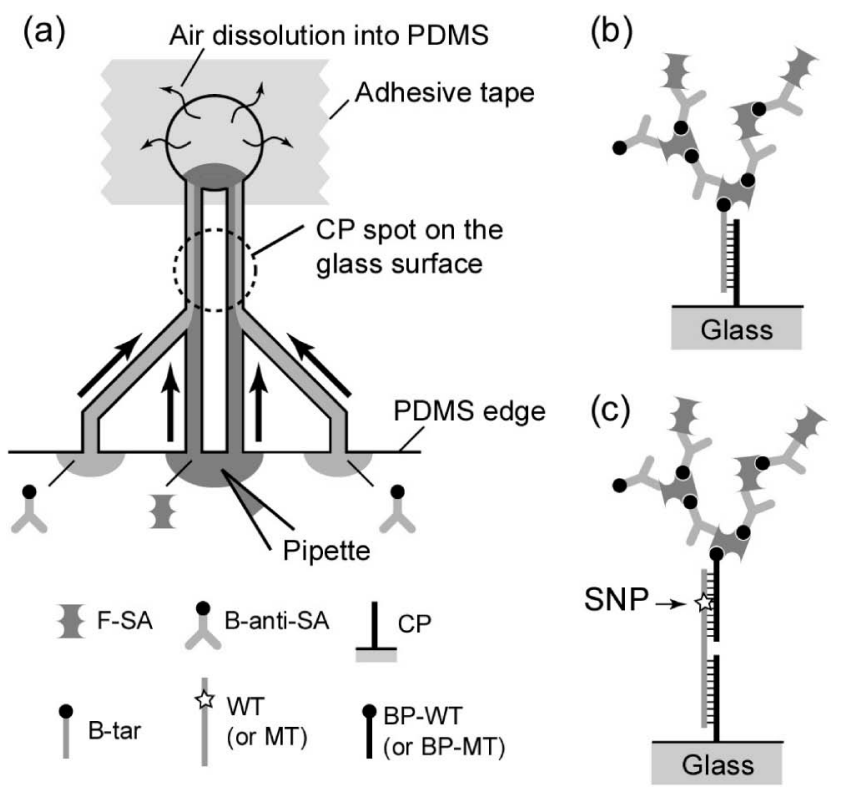

Fig. 2 Schematic representation of the experiments. (a) The reagents dispensed at the inlets are pulled from the waste reservoir, where the dissolution of air into PDMS creates vacuum. The center inlets are always used for one reagent at a time. This picture represents the LFDA step. (b) Direct detection of the biotinylated target. (c) Detection of SNP in the unlabeled target by sandwich hybridization.

hybridization (Fig. 2c). For both experiments, the microchips were identical, and their uses were almost the same. Figure $2 \mathrm{a}$ depicts the LFDA step as an example showing how to use the double-Y microchannels and how power-free pumping works. We adopted this double-Y design to carry out two experiments simultaneously, especially using BP-WT and BP-MT for SNP detection (Fig. 2c). The two halves of the double-Y channels were not completely independent; the center channels were always used for the same solution. The power-free pumping technique was invented by our group. ${ }^{17}$ It is based on the high gas solubility in PDMS. ${ }^{18}$ At atmospheric pressure, PDMS contains a large amount of air, which can be evacuated in a vacuum. When the PDMS is brought back to the atmosphere, air dissolves into the PDMS again. This air dissolution makes a vacuum in the waste reservoir, when the reservoir is sealed with a piece of adhesive tape and the inlets are plugged with the solutions. Therefore, the solutions are pulled by the vacuum in the reservoir. Incidentally, the degassing of the microchip does not need to be performed at the point of analysis, because the degassed microchip can be preserved in a gas-tight, portable container. $^{14}$ In comparison with our previously reported microchip for immunoassay, ${ }^{13}$ the microchip reported here has two major differences: (1) CP was covalently linked to the glass plate, because, unlike antibodies, oligonucleotides cannot be immobilized by physical adsorption stably; and (2) instead of the previous single-Y microchannel, the double-Y design was adopted, as described above.

First, we tried the direct detection of B-tar to check whether the combination of the power-free microchip and the LFDA is effective not only for the immunoassays, ${ }^{13}$ but also for DNA detection. We used the left half of the double-Y channels for the detection of B-tar at various concentrations, while the right half was basically used for the blank sample. All of the samples contained 1.0 $\mu \mathrm{M}$ B-ran, which was at least 100-times as much as B-tar. After blocking the microchannels, we injected the two 


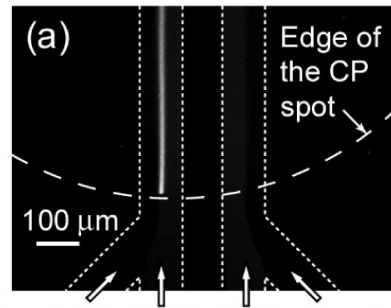

(b)

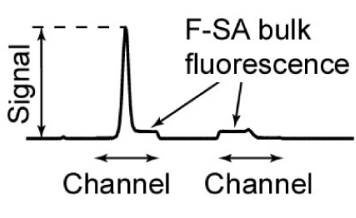

B-anti-SA F-SA F-SA B-anti-SA

Fig. 3 (a) Typical fluorescence image of the microchannels executing the LFDA. The B-tar concentrations were $0.10 \mathrm{nM}$ and 0 for the left and the right channels, respectively. The LFDA time was 10 min. (b) Intensity profile plot across the microchannels.

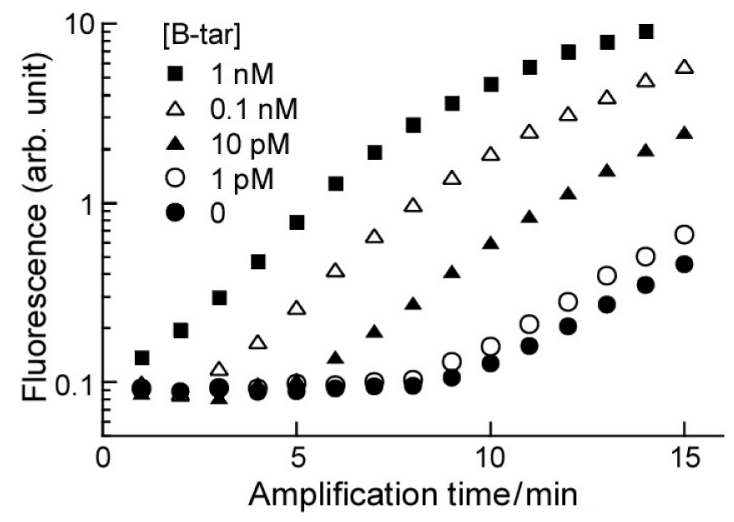

Fig. 4 Time course of the peak fluorescence intensities during the LFDA for the direct detection of B-tar.

samples into the left and the right inlets. At the same time, the blocking solution was flowed from the center inlets. All of the solutions were pumped into the microchannels in $5 \mathrm{~min}$. Then, LFDA was started by applying the F-SA and the B-anti-SA solutions at the proper inlets (Fig. 2a). Aggregates of F-SA and $\mathrm{B}$-anti-SA molecules were expected to form on the B-tar molecules that were captured by CP (Fig. 2b).

Figure 3a shows a typical fluorescence image during the LFDA with samples of $0.10 \mathrm{nM} \mathrm{B-tar}$ and blank for the left and the right channels, respectively. A bright line appeared at the expected position: (1) it appeared only in the left channel where B-tar had been supplied, (2) it appeared only on the CP spot where B-tar should have been captured, and (3) it appeared in the middle of the channel where F-SA and B-anti-SA were supplied from the two laminar streams. This image proves that the assay design shown in Fig. $2 b$ was successfully realized. A fluorescence intensity profile plot across the microchannels is shown in Fig. $3 b$. In this plot, one can see slight rises in intensity in both of the channels. These rises were caused by fluorescence from the bulk F-SA solution. We defined the peak intensity from the background level as the signal, which had a minimum non-zero value caused by the bulk fluorescence.

Figure 4 shows the time course of signals generated by the LFDA for the detection of B-tar at various concentrations. The signals began to rise from the minimum value (F-SA bulk fluorescence) at some time, depending on the B-tar concentrations. Namely, a higher concentration caused an earlier rise. The blank sample showed the latest rise, which was probably initiated by nonspecific adsorption of F-SA or $\mathrm{B}$-anti-SA. Once the signals rose, they exponentially grew at

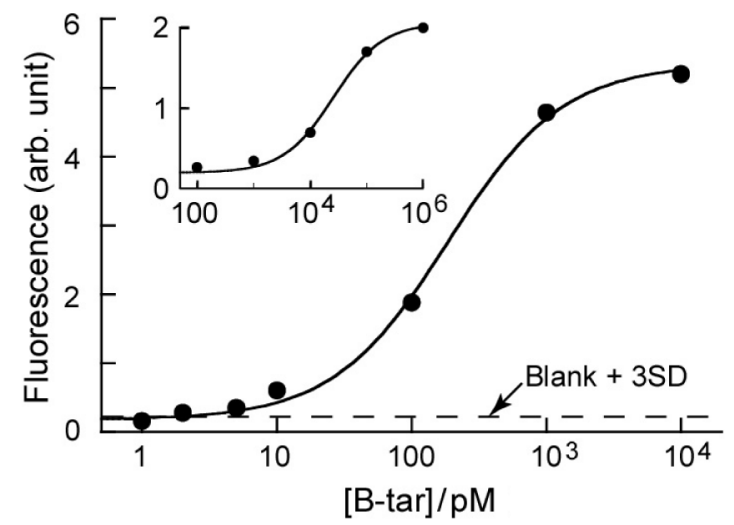

Fig. 5 Calibration plot of the direct detection of B-tar with $10 \mathrm{~min}$ LFDA. The curve was obtained by fitting the data points with Eq. (1). The inset shows the calibration plot of the control experiment without the LFDA.

similar rates. As a result, higher signals were obtained with higher B-tar concentrations. The coefficient of variation of the signal from the blank sample ranged from $5 \%$ (at $3 \mathrm{~min}$ ) to $19 \%$ (at $11 \mathrm{~min})(n=7$, measured with different microchips). The other samples were measured only once.

Figure 5 shows a calibration plot of the direct detection of B-tar with the LFDA time for $10 \mathrm{~min}$. The data points were fitted with the four-parameter logistic function: ${ }^{19}$

$$
y=d+(a-d) /\left[1+(x / c)^{\mathrm{b}}\right]
$$

where $a, b, c$, and $d$ are fitting parameters. The LOD was calculated as $x$, which gives $y$ different from the blank signal by 3SD. As a result, we obtained an LOD of $2.2 \mathrm{pM}$. This LOD was lower than those in most of the previous reports concerning solid-phase hybridization assays on microchips. ${ }^{6-12}$ In fact, the LOD values of these reports fell within the range from $19 \mathrm{pM}^{8}$ to $1 \mathrm{nM},{ }^{6}$ with one exception $\left(9 \mathrm{fM}^{9}\right)$. The detection time of our microchip, $20 \mathrm{~min}$, was roughly the average of those in previous reports: $10 \min ^{6}$ to $30 \mathrm{~min} .{ }^{9}$

We carried out a set of control experiments without the LFDA. Instead of the LFDA step (Step 3 in the Experimental section), we injected the F-SA solution into all of the inlets $(0.5,1.0$, and $0.5 \mu \mathrm{L}$ for the left, the center, and the right inlets, respectively). We then washed the channels with the blocking solution. The results are shown in the inset of Fig. 5. In this case, the LOD was calculated to be $2.7 \mathrm{nM}$. This indicated that the LFDA had improved the LOD by 3 orders of magnitude. In our previous work, ${ }^{13}$ the LFDA had also improved the LOD of immunoassay by 3 orders of magnitude. Although this exact agreement of the extents of improvements might be just a coincidence, it can be concluded that the combination of the power-free microchip and the LFDA is very effective for DNA detection as well as immunoassays.

Having confirmed the effectiveness of our method for the detection of biotinylated DNA, we tried SNP detection of unlabeled DNA by sandwich hybridization (Fig. 2c). In this set of experiments, the left half of the double-Y microchannels was used for the hybridization of BP-WT to the target DNA, while the right half was used for the hybridization of BP-MT. Specifically, we injected BP-WT, the target, and BP-MT into the left, the center, and the right inlets, respectively. In other words, two sandwich hybridization complexes were formed in a single step. Then, LFDA was started in the same way as that for the 


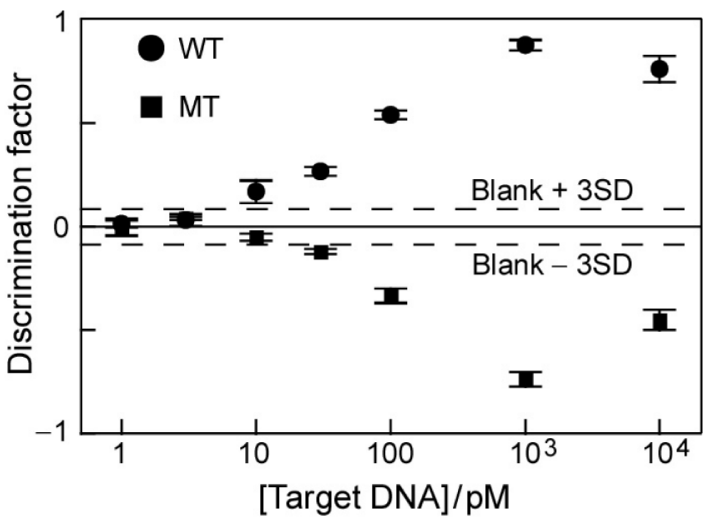

Fig. 6 Calibration plot of the SNP detection by the sandwich hybridization with $10 \mathrm{~min}$ LFDA. The discrimination factor is defined as Eq. (2). The error bars indicate $\pm 1 \mathrm{SD}(n=3$, measured with different microchips).

B-tar detection described above. After $10 \mathrm{~min}$ of LFDA, the fluorescence signals in both channels were measured. From the signal values, the discrimination factor (DF) was calculated by the following equation: ${ }^{3}$

$$
\mathrm{DF}=\left(S_{\mathrm{W}}-S_{\mathrm{M}}\right) /\left(S_{\mathrm{W}}+S_{\mathrm{M}}\right)
$$

where $S_{\mathrm{W}}$ and $S_{\mathrm{M}}$ denote the signals of the left and right channels, respectively. The ideal DF values for the WT and MT targets are 1 and -1 , respectively.

The DF values are plotted against the target concentrations in Fig. 6. For both WT and MT, the absolute values of DF increased along with the elevating concentrations up to $1.0 \mathrm{nM}$, and then somewhat decreased at $10 \mathrm{nM}$. This decrease was probably caused by the combination of two effects: signal saturation of the complementary probe and nonspecific hybridization of the mismatched probe. At lower concentrations, WT and MT showed slightly different behaviors. With the 3SD criterion, $10 \mathrm{pM}$ WT was detectable, whereas MT at the same concentration was not. MT became detectable at $30 \mathrm{pM}$ or higher concentrations. This difference was probably caused by different $T_{\mathrm{m}}$ values of the biotinylated probes, as indicated in Table 1 . These LOD values, 10 and $30 \mathrm{pM}$, were considerably worse than that of B-tar, $2.2 \mathrm{pM}$. However, this seems to be a reasonable compromise because we raised the assay specificity for determining the SNP, and the LOD values were still much lower than the typical concentration of the PCR products: submicromolar level. ${ }^{7}$ Here, we tested only homozygous samples. For practical applications to SNP genotyping of diploid species, like humans, heterozygous samples should also be evaluated.

\section{Conclusions}

We have proven that the combination of the power-free microchip and the LFDA is effective for the simple, rapid, and sensitive detection of DNA. Although the proposed method is promising for POCT applications, there still remain at least two issues to be improved. First, since we tested only a single CP spot, an extension of this work toward multiple CP spots should be explored. The patterning technique making CP lines perpendicular to the microchannels ${ }^{6,11}$ seems to be easier to implement than the conventional spotting techniques, because the former does not need any precise alignment between the $\mathrm{CP}$ patterns and the microchannels. Second, although we achieved the LOD in the picomolar range, this is still insufficient for the detection of genomic DNA without PCR. In fact, PCR-less detection was reported only with ultrasensitive devices with LOD in the femtomolar range.,9 For an improvement of the LOD, the suppression of nonspecific adsorption must be further investigated. At the same time, the use of peptide nucleic acid (PNA) for the probes may be effective, because, in general, PNA probes are known to have better affinity and specificity than DNA counterparts. ${ }^{20}$

\section{References}

1. C. Syvanen, Nat. Rev. Genet., 2001, 2, 930.

2. J. Perkel, Nat. Methods, 2008, 5, 447.

3. Y. P. Bao, M. Huber, T. F. Wei, S. S. Marla, J. J. Storhoff, and U. R. Muller, Nucleic Acids Res., 2005, 33, e15.

4. D. Erickson and D. Q. Li, Anal. Chim. Acta, 2004, 507, 11.

5. N. Lion, F. Reymond, H. H. Girault, and J. S. Rossier, Curr. Opin. Biotechnol., 2004, 15, 31.

6. L. Wang and P. C. H. Li, J. Agric. Food Chem., 2007, 55, 10509.

7. K. Hatakeyama, T. Tanaka, M. Sawaguchi, A. Iwadate, Y. Mizutani, K. Sasaki, N. Tateishi, and T. Matsunaga, Lab Chip, 2009, 9, 1052.

8. W. Wei, J. Y. Cheng, C. T. Huang, M. H. Yen, and T. H. Young, Nucleic Acids Res., 2005, 33, e78.

9. S. P. Mulvaney, C. N. Ibe, C. R. Tamanaha, and L. J. Whitman, Anal. Biochem., 2009, 392, 139.

10. Erickson, X. Z. Liu, R. Venditti, D. Q. Li, and U. J. Krull, Anal. Chem., 2005, 77, 4000.

11. H. Chen, L. Wang, and P. C. H. Li, Lab Chip, 2008, 8, 826.

12. G. Y. Jia, K. S. Ma, J. Kim, J. V. Zoval, R. Peytavi, M. G. Bergeron, and M. J. Madou, Sens. Actuators, B, 2006, 114 , 173.

13. K. Hosokawa, M. Omata, and M. Maeda, Anal. Chem., 2007, 79, 6000 .

14. K. Hosokawa, M. Omata, K. Sato, and M. Maeda, Lab Chip, 2006, 6, 236.

15. A. Inoue, A. Han, K. Makino, K. Hosokawa, and M. Maeda, Lab Chip, 2009, 9, 3297.

16. J. B. Lamture, K. L. Beattie, B. E. Burke, M. D. Eggers, D. J. Ehrlich, R. Fowler, M. A. Hollis, B. B. Kosicki, R. K. Reich, S. R. Smith, R. S. Varma, and M. E. Hogan, Nucleic Acids Res., 1994, 22, 2121.

17. K. Hosokawa, K. Sato, N. Ichikawa, and M. Maeda, Lab Chip, 2004, 4, 181.

18. T. C. Merkel, V. I. Bondar, K. Nagai, B. D. Freeman, and I. Pinnau, J. Polym. Sci., Part B: Polym. Phys., 2000, 38, 415.

19. P. Diamandis and T. K. Christopoulos, "Immunoassay", 1996, Academic Press, San Diego.

20. J. Weiler, H. Gausepohl, N. Hauser, O. N. Jensen, and J. D. Hoheisel, Nucleic Acids Res., 1997, 25, 2792. 